mean daily incidences of bacteriuria were similar for patients whose catheters were irrigated and those in which no irrigation was used. More frequent disconnections of the catheter-tubing junctions that allowed entry of organisms into the bladder were thought to have neutralized any beneficial effect of bacterial suppression in the irrigated drainage systems. In addition, bacterial isolates that were recovered from urine cultures of the irrigated systems contained a greater percentage of antibiotic-resistant organisms than isolates from non-irrigated systems. This group of investigators concluded that, on the basis of their findings, bladder irrigation using a continuous infusion of an antimicrobic solution should not be recommended. The Centers for Disease Control's guidelines for the prevention of catheter-associated urinary tract infections endorse this point of view, stating "Continuous irrigation of the bladder has not proven to be useful and should not be performed as a routine infection prevention measure (moderately recommended)."2

1. Warren JW, Platt R, Thomas RJ, Rosner B, Kass EH: Antibiotic irrigation and catheter-associated urinary-tract infections. N Engl J Med 1978; 299:570-573.

2. CDC Guideline for Prevention of Catheter-Associated Urinary Tract Infections. Infect Control 1981; 2:125-130.

Richard A. Garibaldi, MD Professor of Medicine Division of Infectious Diseases University of Connecticut Health Center Farmington, Connecticut

\section{The Susceptibility of Health Care Workers to Varicella-Zoster Virus}

\section{To the Editor:}

Varicella-zoster infection in hospital personnel continues to concern us, as well as other health care workers (Gurevich, Infect Control 1986; 7:393). Use of an immunization certificate requirement, however, presents a solution to only a portion of the problem.

Recently, a nurse caring for a normal patient (nonimmunocompromised), with localized herpes zoster infection developed a severe varicella infection. The index case had been cared for using disease-specific isolation guidelines as outlined in CDC criteria. Since his personal hygiene was adequate, private room and mask were not required nor were they used. Twelve days after her initial contact with the infected patient she noted her first vesicle. Subsequently, she was hospitalized with disseminated varicella infection.

Employee health records showed that she had been treated for viral hepatitis in another community several years prior and had been maintained, until recently, on oral prednisone therapy. The nurse neglected to share with our employee health nurse the fact that she had had long-term steroid therapy and that it had been stopped only recently. In addition, she also neglected to relate that she had been tested for the presence of varicella antibody two years ago and had been found negative.

As a result of this occurrence, we have not only adopted a new immunization certification program, but also are reviewing employee applicant health records to determine past medical illnesses and medical therapy as they relate to immune competence. We cannot assume that employees recognize the importance of reporting past medical experiences. More importantly, not everyone is correctly aware of their viral exanthem history and may unknowingly assume immunity, when none exists.

Sharing our experience with others may prevent similar occurrences in other health care facilities which care for patients with herpes zoster infection.

Stephen R. Zellner, MD Chairman, Infection Control Committee Fort Myers Community Hospital Fort Myers, Florida

\section{To the Editor:}

We have been using the CDC Guideline for Isolation Precautions in Hospitals since its release in July, 1983. In normal individuals, precautions for the transmission of varicella-zoster virus include the use of a private room only when the patient's personal hygiene is poor. Gloves are recommended when it becomes necessary to touch infective material.

Although airborne spread of the varicella-zoster virus is recognized to occur in hospitals, precautions do not indicate private room confinement in every case. We recently had experience with the spread of herpes zoster infection from an infected patient to a susceptible individual across the hall that is probably the result of the airborne route. The patient who became infected was recuperating from a motor vehicle accident and had been confined in his room for several weeks. The zoster patient was admitted because of her inability to care for herself at home. Other than for her zoster infection, she was in good medical health. Not only did the traumatized patient develop varicella infection, but two employees caring for him during his confinement period also developed the infection. They did not have contact with the index case.

Perhaps the guidelines as stated should be re-evaluated to include private, well-ventilated rooms for all patients with herpes zoster infection regardless of their personal hygiene status and extent of infection. Since not all patients admitted to hospitals today have immunity to the varicellazoster virus, we should consider all patients susceptible and use appropriate precautions when caring for the already infected patient.

Health care workers have unfortunately developed a complacent attitude when caring for the zoster infected patient. Further in-service education in the management of patients with herpes zoster infection may help prevent professional staff exposure and infection.

It is this approach to the infected patient that may well allow for contact and/or airborne spread of the varicella-zoster virus.

Stephen Zellner, MD Nancy Polley, RN, BA, CIC Epi-Systematics, Inc., Consultants Fort Myers, Florida 